\title{
Late-Onset, Noninfectious Pulmonary Complications following Allogeneic Hematopoietic Stem Cell Transplantation: A Nationwide Cohort Study of Long-Term Survivors
}

\author{
Ole Henrik Myrdal ${ }^{a, b}$ Trond Mogens Aaløkken ${ }^{b, c}$ Phoi Phoi Diep ${ }^{b, d, e}$

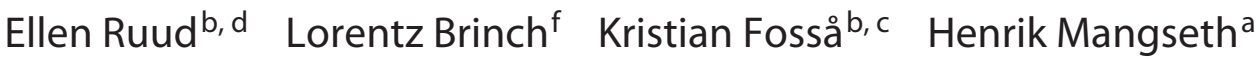 \\ Johny Kongerud ${ }^{a, b}$ Liv Ingunn Sikkeland ${ }^{a, b}$ May B. Lund ${ }^{a, b}$

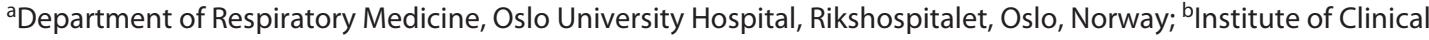
Medicine, University of Oslo, Oslo, Norway; ' Department of Radiology, Oslo University Hospital, Oslo, Norway; ${ }^{\mathrm{d} D e p a r t m e n t}$ of Pediatric Oncology and Haematology, Oslo University Hospital, Rikshospitalet, Oslo, Norway; ${ }^{\text {eDivision }}$ of Pediatric and Adolescent Medicine, Department of Pediatric Research, Oslo University Hospital, Oslo, Norway;
\end{abstract} \\ fDepartment of Haematology, Oslo University Hospital, Rikshospitalet, Oslo, Norway
}

\section{Keywords}

Allogeneic hematopoietic stem cell transplantation .

Bronchiolitis obliterans syndrome · High-resolution CT .

Pulmonary function · Long-term follow-up

\begin{abstract}
Background: Survivors of allogeneic hematopoietic stem cell transplantation (allo-HSCT) are at risk for pulmonary adverse events. Data on late-onset noninfectious pulmonary complications in long-term adult survivors of allo-HSCT are limited and incomplete. Objectives: This study aimed (1) to determine occurrence and degree of pulmonary sequelae in adult survivors of allo-HSCT and (2) to identify associations between pulmonary function, high-resolution CT (HRCT), and clinical characteristics. Method: In a nationwide, singlecenter cross-sectional study, 103 survivors (aged median [range] 35 [17-58] years, 53\% females) were examined 17 (6-32) years after allo-HSCT and compared with healthy controls $(n=105)$. Methods included pulmonary function tests and HRCT. Results: Chronic graft-versus-host disease was di-
\end{abstract}

agnosed in $33 \%$ of survivors, including $12 \%$ with bronchiolitis obliterans syndrome (BOS). Mean lung volumes (TLC, $\mathrm{FVC}$, and $\mathrm{FEV}_{1}$ ) and gas diffusing capacity were $>80 \%$ of predicted for the survivors as a group, but significantly lower than in healthy controls. Pathological HRCT findings were detected in $48 \%$ of the survivors (71\% airways disease, $35 \%$ interstitial lung disease, and $24 \%$ apical subpleural interstitial thickening). Air trapping (\%) on HRCT correlated with \% predicted $\mathrm{FEV}_{1}, p<0.001$. In a multiple logistic regression model, both BOS and pathological findings on HRCT were associated with chemotherapy prior to allo-HSCT, $p<0.05$. Conclusions: Long-term allo-HSCT survivors had significantly lower pulmonary function than age- and gender-matched healthy controls and nearly half had pathological findings on HRCT. Longitudinal data will determine if pulmonary sequelae will remain stable or progress. We recommend lifelong monitoring of pulmonary function in allo-HSCT survivors. HRCT provides additional information, but is not suited for surveillance.

(c) 2021 The Author(s) Published by S. Karger AG, Basel
(C) 2021 The Author(s)

Published by S. Karger AG, Basel

This is an Open Access article licensed under the Creative Commons Attribution-NonCommercial-4.0 International License (CC BY-NC) (http://www.karger.com/Services/OpenAccessLicense), applicable to the online version of the article only. Usage and distribution for commercial purposes requires written permission.
Correspondence to:

Ole Henrik Myrdal, omyrda @ous-hf.no 


\section{Introduction}

Allogeneic hematopoietic stem cell transplantation (allo-HSCT) is an established, life-saving treatment for a diversity of malignant and nonmalignant disorders [1]. It is, however, associated with numerous adverse effects that may complicate long-term outcomes [2].

Pulmonary complications have been reported in onethird of allo-HSCT recipients, affecting morbidity and mortality [3]. Factors associated with pulmonary complications are graft-versus-host disease (GVHD), conditioning regimen, underlying lung disease, and factors related to treatment prior to allo-HSCT [3]. In brief, pulmonary complications may develop due to either infectious or noninfectious causes. Late-onset noninfectious pulmonary complications (LONIPCs) may be caused by chemotherapy used in the myelosuppressive conditioning regimen [4], total body irradiation $[5,6]$, or as an immunological response induced by the graft [7]. The latter is typically lateoccurring in the form of chronic GVHD (cGVHD) [8]. The most frequent LONIPC is bronchiolitis obliterans, which is linked to cGVHD [9]. Bronchiolitis obliterans is diagnosed by its histological pattern of fibrogenic deposition in small airways. A lung biopsy is needed, which requires an invasive and potentially harmful procedure. Therefore, the clinically based diagnosis bronchiolitis obliterans syndrome (BOS) is usually preferred. Criteria for the diagnosis of $\mathrm{CGVHD}$ and BOS have been published by the National Institutes of Health (NIH) [10]. The diagnosis of BOS is primarily based on pulmonary function tests with supporting criteria based on high-resolution computed tomography (HRCT) and differs from the diagnosis of BOS following lung transplantation [11]. Also, interstitial lung diseases (ILDs) may occur in survivors of allo-HSCT and are often labeled LONIPCs as well [9]. The ILDs have different appearances and histological findings [12]. One is pleuroparenchymal fibroelastosis which is characterized by elastic fibrosis, involving lung tissue in the upper lobes [13]. Hence, the development of LONIPCs is complex, with a range of rare conditions from ILDs to BOS, and there may also be an overlap between these disorders in the same patient [3]. To our knowledge, HRCT and pulmonary function tests have been applied jointly in only a few clinical studies with focus on late complications in adult allo-HSCT survivors [14-16]. One was a large $(n=198)$, prospective study, median 6 years after allo-HSCT, reporting 44 survivors with LONIPCs, mainly in terms of BOS and ILD [14]. Other studies applying HRCT have been restricted to patients with previously diagnosed cGVHD/ BOS [15-17]. Hence, little is known of LONIPCs in adult

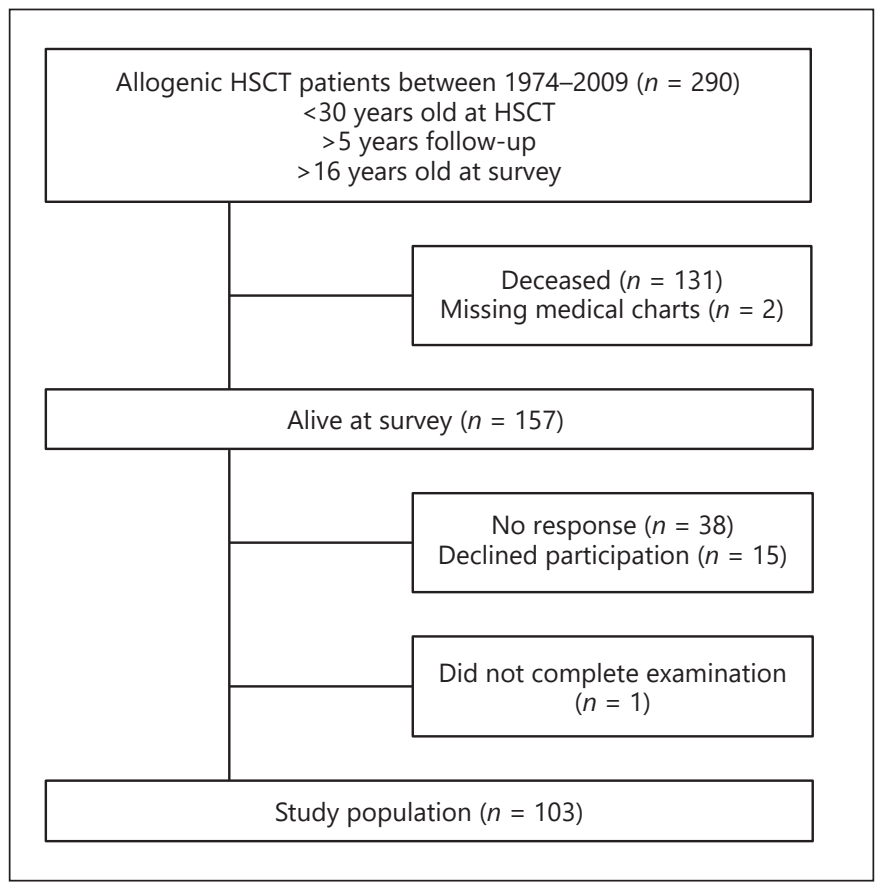

Fig. 1. Study selection of long-term survivors after allo-HSCT. Allo-HSCT, allogeneic hematopoietic stem cell transplantation.

allo-HSCT survivors with observation periods exceeding 1 decade. The present study was carried out within the context of a large and comprehensive project investigating health conditions in young, very long-term survivors of allo-HSCT [18-21]. All survivors were examined with both HRCT and pulmonary function tests. We aimed to assess the occurrence and degree of late pulmonary sequelae in a nationwide cohort of survivors who had been treated with allo-HSCT in childhood, adolescence, or early adulthood median 17 years previously. We also aimed to identify associations between clinical characteristics, HRCT findings, and pulmonary function.

\section{Materials and Methods}

\section{Design and Study Population}

The study was conducted at Oslo University Hospital from August 2014 to February 2016, as a part of a single-center, nationwide cross-sectional study covering a broad range of late treatment-related effects after allo-HSCT [18-21]. The survivors were eligible for inclusion if they were younger than 30 years at transplantation, older than 16 years at examination, and had minimum 5 years of observation $(n=157)$ (Fig. 1). All participating survivors $(n=103)$ gave their written informed consent. The study was approved by the South-East Regional Committee for Medical and Health Research Ethics (2014/370). 


\section{Healthy Controls}

For pulmonary function testing, 105 healthy, never-smoking subjects (age [median, range] 35 [20-59] years, 56\% females) with no history of cancer or pulmonary disease were recruited among university and hospital employees through local advertisements. The controls did not undergo HRCT examination since it was considered unethical to expose healthy subjects to irradiation.

\section{Clinical Assessment}

Clinical data, including gender, age, BMI, smoking habits, medical history, physician-diagnosed lung disease, and current medication, were recorded. Routine blood tests included hemoglobin $(\mathrm{Hb})$ levels.

\section{Pulmonary Function}

Measures of pulmonary function were performed according to the American Thoracic Society/European Respiratory Society (ATS/ERS) guidelines $[22,23]$ and included total lung capacity (TLC), vital capacity (VC), residual volume (RV), forced vital capacity (FVC), forced expiratory volume in $1 \mathrm{~s}\left(\mathrm{FEV}_{1}\right)$, and diffusing capacity of the lung for carbon monoxide $\left(\mathrm{DL}_{\mathrm{CO}}\right)$. $\mathrm{DL}_{\mathrm{CO}}$ values were also corrected for $\mathrm{Hb}$ levels, obtained on the same day as pulmonary function testing. Since $\mathrm{Hb}$ correction of $\mathrm{DL}_{\mathrm{CO}}$ did not significantly influence the results of the analyses, the variable $\mathrm{DL}_{\mathrm{CO}}$ $\mathrm{Hb}$ was not reported in the Results section. The predicted values for spirometry and $\mathrm{DL}_{\mathrm{CO}}$ were taken from the Global Lung Initiative $[24,25]$ and static volumes from the European Community for Steel and Coal [26]. All tests were performed with the Jaeger Master Screen Body (Eric Jaeger, Wurzburg, Germany). BOS was diagnosed according to NIH criteria [10]: (1) new-onset airway obstruction with $\mathrm{FEV}_{1} / \mathrm{FVC}<0.70$, and (2) $\mathrm{FEV}_{1}<75 \%$ of predicted, with $10 \%$ decline over $<2$ years, and (3) absence of infection, and (4) one of 2 supportive features of air trapping, either on expiratory HRCT or by residual volume (RV) $>120 \%$ of predicted. Due to the cross-sectional study design, we cannot tell if airway obstruction was new-onset or not, or if $\mathrm{FEV}_{1}$ had declined $>10 \%$ in 2 years. Restrictive impairment and impaired gas diffusing capacity were defined as, respectively, TLC and $\mathrm{DL}_{\mathrm{CO}}<80 \%$ of predicted. We calculated the lower limit of normal in order to confirm that the cutoff $80 \%$ predicted fitted the lower 5 th percentile of the reference values [27]. We used the term pulmonary sequelae for describing the combination of impairments in pulmonary function and pathological findings on HRCT.

\section{Acquisition and Review of HRCT Images}

CT examinations were performed with a LightSpeed 16 scanner (GE Healthcare, Milwaukee, WI, USA) and obtained in the supine position, during deep inspiration and breath-holding. Tube current settings were adjusted to each patient's weight, but with low dose references (noise index: 40). No intravenous contrast material was used. In all subjects, supplementary expiratory scans were obtained with $1.25-\mathrm{mm}$ section thickness at $10-\mathrm{mm}$ intervals. Supplementary prone position scans were obtained when considered necessary to differentiate between fine reticular fibrosis and dependent atelectasis. The images were reviewed on a PACS (Picture Archiving and Communication System) screen in random order and in consensus by 2 experienced chest radiologists (T.M.A. and K.F.) and 1 pulmonologist (O.H.M.) with special training in interpretation of HRCTs. The reviewers were blinded to lung function and clinical data. The presence, extent, and distribution of interstitial findings

Table 1. Characteristics of 103 long-term survivors of allo-HSCT

\section{Male/female}

Age at transplantation, median (range)

Age at follow-up, median (range)

Years of observation, median (range)

BMl, median (range), $\mathrm{kg} / \mathrm{m}^{2}$

Smoking

Never

Former

Daily

Pack-years, median (range)

Physician-diagnosed asthma

Underlying diagnosis

Benign hematological disorders

Immunodeficiencies and metabolic disorders

Malignancy

Acute myeloid leukemia

Chronic myeloid leukemia

Acute lymphoblastic leukemia

Others

Pre allo-HSCT treatment and conditioning Intravenous chemotherapy for malignancies Chemotherapy, busulfan/cyclophosphamide Chemotherapy + total body irradiation None

Donor

Matched related donor $65(63)$

Haploidentical donor

Matched unrelated donor

Gender mismatch

Graft-versus-host disease

Acute, grade II-IV

Chronic

Data are presented as median (range) or $n$ (\%). Allo-HSCT, allogeneic hematopoietic stem cell transplantation; pack-years, the number of packs with 20 cigarettes smoked/day, multiplied by years of consumption; BMI, body mass index.

were evaluated according to the HRCT criteria of ILD recommended by the Nomenclature Committee of the Fleischner Society [28]. HRCT-detected ILD was defined as reticular pattern and/or ground glass opacities. Airways disease was defined as bronchiectasis, and/ or air trapping, and/or mosaic pattern, and/or centrilobular micronodules. In the setting of long-term survivors of allo-HSCT with the risk of LONIPCs, the HRCT findings were categorized into 3 major groups: airways disease, apical irregular subpleural interstitial thickening, and other signs of ILD. The distribution of pathology was reviewed in 4 zones: (a) above the aortic arch, (b) between the aortic arch and the level of the carina, (c) between the level of the carina and the level of the inferior pulmonary veins, and (d) below the inferior pulmonary veins. The extent of involvement of pathological findings was evaluated for each lung zone. The severity of bronchiectasis was scored either as bronchial wall thickening without distinct ectasis or bronchiectasis in localizations $(\mathrm{a}-\mathrm{d})$. The extent of air trapping in each zone was assigned a score based on the percentage of lung parenchyma involved, with an overall score 
Fig. 2. Pulmonary function in 103 longterm survivors of allo-HSCT and 105 healthy controls. Comparison between controls and allo-HSCT w/o BOS. Data presented as $\%$ predicted (mean $[95 \% \mathrm{CI}]$ ). Allo-HSCT, allogeneic hematopoietic stem cell transplantation; BOS, bronchiolitis obliterans syndrome.

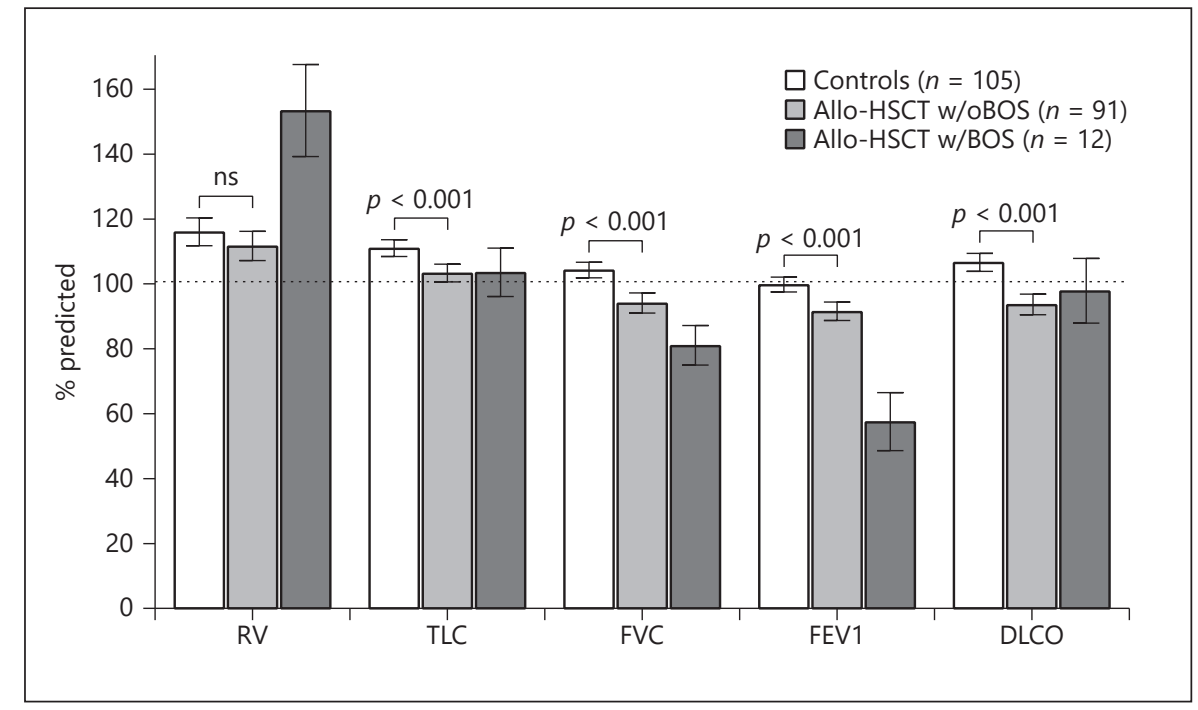

of involvement for each patient derived by summing the scores of the 4 CT levels. Subsegmental air trapping comprising $<5 \%$ of the lung parenchyma was considered normal [27].

\section{Statistical Analysis}

Student's $t$ test or Mann Whitney U test was used, as appropriate, to compare continuous data between groups. $\chi^{2}$ test or Fisher's exact test was used to compare categorical variables. Univariate and multiple logistic regression analyses were used to detect associations between dependent variables and relevant covariates, controlling for extraneous effects. The independent variables which entered the regression models were those hypothesized a priori for biological or clinical reasons or found to be significant at a $20 \%$ level by univariate analysis. Pulmonary function in patients with BOS was analyzed separately since the diagnostic criteria for BOS are based on such tests [10]. A 2 -sided $p$ value $<0.05$ was considered significant. Standard statistical analyses were performed with SPSS software (IBM SPSS statistics, version 26).

\section{Results}

A total of 157 survivors fulfilled the inclusion criteria and 103 (66\%) participated in the study (Fig. 1). The 54 nonparticipating survivors included more males than females ( $69 \%$ vs. $47 \%, p=0.01$ ), were (median [range] years) younger at the time of allo-HSCT $14(0.8-30)$ versus 20 $(0.3-30), p=0.02$, and had shorter observation time 12 (5$24)$ versus 17 (6-32), $p<0.001$ than the included survivors. The nonresponders were comparable to the included survivors with respect to diagnosis prior to transplantation.

\section{Patient Characteristics}

Clinical characteristics are outlined in Table 1. Hematological malignancies comprised the underlying diagno- sis in $77(75 \%)$ survivors of whom $46(60 \%)$ had received chemotherapy prior to allo-HSCT. Patients with chronic myeloid leukemia had not received chemotherapy routinely, in contrast to those with other malignancies. Myeloablative regimens with cyclophosphamide/busulfan or cyclophosphamide had been applied in 101 (98\%) survivors. Only 7 subjects had been treated with total body irradiation. Chronic GVHD was diagnosed in 34 (33\%) survivors. Survivors with cGVHD were comparable to those without cGVHD with respect to gender, age, observation time, BMI, and smoking habits. Among subjects with benign underlying disorders $(n=26), 4(15 \%)$ had developed cGVHD compared to 30 (39\%) among those with malignant disorders $(n=77)(p=0.03)$. Chronic GVHD was associated with chemotherapy prior to alloHSCT $(p=0.01)$ and history of acute GVHD $(p=0.001)$, but not with donor match. Mean (SD) Hb was 15.3 (1.0) for males and $13.6(0.9)$ for females.

\section{Pulmonary Function}

Pulmonary function for the allo-HSCT survivors and the healthy controls is shown in Figure 2. Twelve survivors were diagnosed with BOS. They had increased RV and reduced FVC and $\mathrm{FEV}_{1}$, hence confirming that they met the NIH criteria. The survivors without BOS had significantly lower TLC, FVC, $\mathrm{FEV}_{1}$, and $\mathrm{DL}_{\mathrm{CO}}$ than the healthy controls, whereas RV was comparable. Among the allo-HSCT survivors, $4 \%$ had restrictive impairment and $17 \%$ had impaired gas diffusing capacity. No case of impairment was observed among the healthy controls. Twelve of the 13 survivors with asthma had been diagnosed after HSCT, and 4 of them had BOS. Ever-smokers 

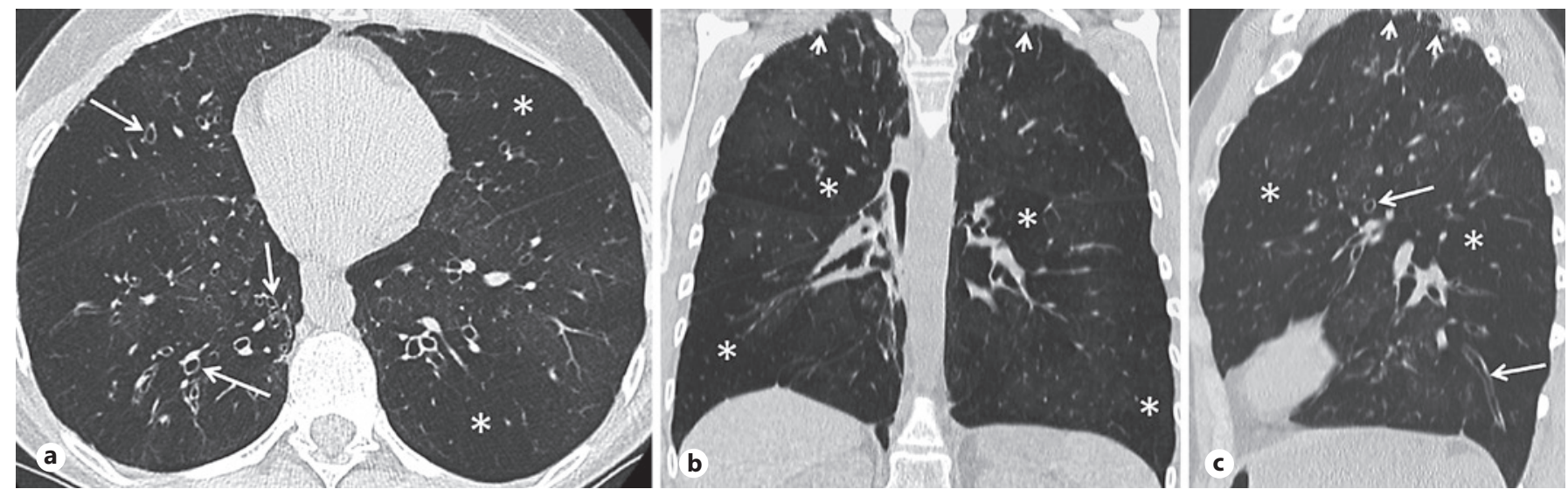

Fig. 3. Axial (a), coronal (b), and sagittal (c) inspiratory HRCT images in a long-term survivor of allo-HSCT with BOS shows airways disease with bronchiectasis (arrows) and mosaic pattern, with heterogeneous lung attenuation comprising geographic areas of decreased attenuation $\left(^{*}\right)$ adjacent to the normal lung. In addition, there is irregular subpleural apical interstitial thickening (arrowheads). Allo-HSCT, allogeneic hematopoietic stem cell transplantation; BOS, bronchiolitis obliterans syndrome; HRCT, high-resolution computed tomography.

Table 2. Pulmonary function in 103 long-term survivors of allo-HSCT according to findings on HRCT

\begin{tabular}{|c|c|c|c|c|c|c|c|c|c|}
\hline & \multirow{2}{*}{$\begin{array}{l}\text { Survivors w/ } \\
\text { normal findings }\end{array}$} & \multirow{2}{*}{$\begin{array}{l}\text { Survivors w/ } \\
\text { pathological } \\
\text { findings }\end{array}$} & \multirow[t]{2}{*}{$p$ value } & \multicolumn{6}{|c|}{ Types of pathological findings $(n=64)$} \\
\hline & & & & airways disease & $\begin{array}{l}p \\
\text { value }\end{array}$ & $\begin{array}{l}\text { interstitial lung } \\
\text { disease } \\
(n=17)\end{array}$ & $p$ value & $\begin{array}{l}\text { apical } \\
\text { subpleural } \\
\text { thickening } \\
(n=12)\end{array}$ & $\begin{array}{l}p \\
\text { value }\end{array}$ \\
\hline TLC & $105(102-109)$ & $102(98-106)$ & 0.13 & $102(97-07)$ & 0.30 & $99(92-106)$ & 0.07 & $102(94-109)$ & 0.33 \\
\hline RV & $112(106-118)$ & $121(113-130)$ & 0.08 & $128(119-139)$ & 0.002 & $116(100-132)$ & 0.57 & $117(102-132)$ & 0.49 \\
\hline FVC & $96(93-100)$ & $90(86-94)$ & 0.06 & $88(83-94)$ & 0.02 & $87(79-95)$ & 0.05 & $89(80-99)$ & 0.20 \\
\hline $\mathrm{FEV}_{1}$ & $94(90-98)$ & $82(76-88)$ & 0.001 & $78(71-85)$ & $<0.001$ & $79(68-91)$ & 0.004 & $82(69-95)$ & 0.03 \\
\hline $\mathrm{FEV}_{1} / \mathrm{FVC}$ & $0.81(0.78-0.82)$ & $0.75(0.71-0.78)$ & 0.002 & $0.72(0.68-0.77)$ & 0.001 & $0.73(0.65-0.81)$ & 0.05 & $0.74(0.65-0.83)$ & 0.02 \\
\hline $\mathrm{DL}_{\mathrm{CO}}$ & $93(88-98)$ & $95(91-100)$ & 0.46 & $96(91-102)$ & 0.31 & $92(84-101)$ & 0.83 & $89(79-100)$ & 0.49 \\
\hline
\end{tabular}

Comparison is between the different findings and normal. One subject may have several findings. Data presented as \% of predicted value $(95 \% \mathrm{CI})$. Allo-HSCT, ALLOGENEIC hematopoietic stem cell transplantation; TLC, total lung capacity in \% of predicted value; RV, residual volume; FVC, forced vital capacity; FEV ${ }_{1}$, forced expiratory volume in $1 \mathrm{~s}$; $\mathrm{DL}_{\mathrm{CO}}$, diffusing capacity for carbon monoxide.

(daily + former) had comparable pulmonary function to never-smokers. There was no significant association between smoking status and BOS. Patients who had received chemotherapy prior to allo-HSCT had lower $\mathrm{DL}_{\mathrm{CO}} \%$ predicted than those who had not received such treatment (mean [95\% CI] \% predicted 78 [73-82] versus 87 [82-91], $p=0.003)$. Of the survivors with impaired $\mathrm{DL}_{\mathrm{CO}}, 2$ had BOS.

\section{HRCT Findings}

HRCT findings are presented in Tables 2 and 3, Figure 3 , and online supplementary Figure 1 (for all online suppl. material, see www.karger.com/doi/10.1159/000520824). Fifty-four (52\%) survivors had normal HRCT (online suppl. Fig. 1a). Among the remaining 49 survivors, 64 pathological HRCT findings were detected (signs of airways disease $n=35$, apical irregular subpleural interstitial thickening $n=12$, and other findings of ILD $n=17$ ). Ten subjects had concomitantly 2 types of pathology and $2 \mathrm{had}$ all 3 types. A picture demonstrating typical CT findings is shown in Figure 3.

Subjects with pathological findings on HRCT had significantly lower $\mathrm{FEV}_{1} \%$ predicted and $\mathrm{FEV}_{1} / \mathrm{FVC}$ than subjects with normal HRCT (supplementary Fig. 1b). All 
Table 3. Odds ratio for selected risk factors related to, respectively, pathological findings on HRCT and BOS in 103 long-term survivors of allo-HSCT

\begin{tabular}{|c|c|c|c|c|c|c|}
\hline \multirow[t]{2}{*}{ Independent variables } & \multicolumn{3}{|c|}{ Pathological findings on HRCT } & \multicolumn{3}{|l|}{ BOS } \\
\hline & odds ratio & $95 \% \mathrm{Cl}$ & $p$ value & odds ratio & $95 \% \mathrm{Cl}$ & $p$ value \\
\hline Female gender & 1.7 & $0.7-3.9$ & 0.205 & 2.3 & $0.6-9.2$ & 0.222 \\
\hline Age at allo-HSCT, years & 1.0 & $1.0-1.1$ & 0.502 & 1.0 & $0.9-1.1$ & 0.970 \\
\hline Observation time, years & 1.0 & $0.9-1.1$ & 0.565 & 0.9 & $0.8-1.0$ & 0.149 \\
\hline Chemotherapy prior to allo-HSCT & 2.4 & $1.0-5.4$ & 0.041 & 4.5 & $1.1-18.6$ & 0.040 \\
\hline
\end{tabular}

Findings on HRCT include signs of airways disease, interstitial lung disease, and apical irregular subpleural, interstitial thickening. Allo-HSCT, allogeneic hematopoietic stem cell transplantation; BOS, bronchiolitis obliterans syndrome.

(except one) of the survivors who fulfilled the pulmonary function criteria for BOS had findings consistent with airways disease. The most frequent single finding on HRCT was air trapping, which was detected in 24 survivors, with mean $29 \%$ of the lung parenchyma involved, ranging from 5 to $77 \%$. The degree of air trapping in $\%$ of lung volume correlated with $\mathrm{RV} \%$ predicted $(r=0.610, p=$ $0.002)$ and inversely with $\mathrm{FEV}_{1} \%$ predicted $(r=-0.771, p$ $<0.001$ ) (online suppl. Fig. 1c). There was no association between pathological findings on HRCT and cGVHD without BOS $(p=0.926)$. Age at allo-HSCT was not a risk factor for pneumological abnormalities in our cohort $(p$ $=0.728$ ). In a multiple logistic regression model, controlling for gender, age at allo-HSCT, and observation time, pathological findings on HRCT and BOS were both associated with chemotherapy prior to allo-HSCT $(p<$ 0.041 and $p<0.040$ ) (Table 3 ).

\section{Discussion}

The main findings of this study were that long-term allo-HSCT survivors had significantly lower pulmonary function than age- and gender-matched healthy controls and nearly half of them had pathological findings on HRCT. Although mean lung volumes (TLC, FVC, and $\mathrm{FEV}_{1}$ ) and gas diffusing capacity were above $80 \%$ predicted for the entire study group, one-third of the survivors had some kind of impairment (17\% impaired gas diffusing capacity, $12 \%$ had developed BOS, and $4 \%$ had restrictive impairment). Survivors who had received chemotherapy prior to allo-HSCT had 2.4-fold and 4.5-fold increased risk for, respectively, pathological findings on HRCT and BOS. The predominant pathological findings on HRCT were signs of airways disease, but also various patterns of ILD, including apical irregular subpleural interstitial thickening suggestive of pleuroparenchymal fibroelastosis, were found. The latter is a rare radiological pattern seen, in particular, after allo-HSCT and lung transplantation [13]. Our findings were indicative of pleuroparenchymal fibroelastosis because upper lobe pleural thickening with associated subpleural fibrosis was present and without involvement of lower lobes. However, detailed radiological features of pleuroparenchymal fibroelastosis are still largely unknown, and definite diagnosis requires histological confirmation [29].

The survivors included in the present study were young adults (median 35 years old), and approximately three-quarters were never-smokers. The former/current smokers had few pack-years (median 3 pack-years) which probably explains why their pulmonary function was comparable to the never-smokers. Four of the 12 survivors with physician-diagnosed asthma after HSCT also had BOS. It is possible that respiratory symptoms caused by BOS may have led to misclassification of asthma.

Pulmonary function was well preserved for the survivors who had not developed BOS. However, compared to the healthy controls, also the survivors without BOS had significantly reduced TLC, FVC, FEV1, and gas diffusing capacity. The latter was reduced, in particular, in subjects who had received intravenous high-dose courses of chemotherapy for malignant blood disorders prior to alloHSCT. This finding is in line with reports from various other studies indicating that $\mathrm{DL}_{\mathrm{CO}}$ is the most sensitive test for detecting chemotherapy-induced lung injury [3035]. In previous studies, we have found impaired gas diffusing capacity in long-term lymphoma survivors after high-dose therapy with autologous stem cell transplantation [33] and in very long-term adult survivors of childhood acute lymphoblastic leukemia [34]. Late adverse ef- 
fects of chemotherapy on gas diffusing capacity have also been shown in patients treated for lung cancer [32] and breast cancer [35]. Although the underlying mechanisms of cytotoxic lung injury are multifactorial and remain unclear, it is thought that microvascular damage may be a common denominator and an important feature [30,31].

Our findings suggest that in combination with pulmonary function tests, HRCT is a useful tool for diagnosing BOS. In the present study, all but one subject with BOS also had pathological findings on HRCT consistent with airways disease. However, signs of airways disease (air trapping, small airway wall thickening, bronchiectasis, and mosaic attenuation) on HRCT are too common and too unspecific to be used alone to diagnose BOS. Air trapping was the single most frequent finding on HRCT in our study and occurred in nearly one-fourth of the survivors of whom only half fulfilled the pulmonary function criteria for BOS. To our knowledge, only one other largesample study has used both HRCT and pulmonary function tests in order to identify long-term noninfectious lung complications in young adult survivors of alloHSCT [14]. A few smaller studies have reported HRCT findings in survivors who had already been diagnosed with airway obstruction by previous pulmonary function testing [15-17]. It is, however, difficult to compare HRCT findings from different studies due to various study designs, definitions, and methods of reporting. Air trapping is defined as retention of air distal to airway obstruction, best visualized on expiratory HRCT scans. It is the most common finding on HRCT suggestive of BOS. We chose to report air trapping as a percentage of lung parenchyma, which is a well-established method [16, 28, 36, 37]. We found a strong correlation between \% air trapping on HRCT and FEV $1 \%$ predicted and RV\% of predicted. This is in agreement with previous studies that have reported similar associations between airflow obstruction and air trapping in comparable allo-HSCT study populations [15-17]. The prevalence of BOS was $12 \%$ in our study, which is comparable to $11 \%$ reported by Bergeron et al. [14]. This is interesting, given the difference in follow-up between the 2 studies (median 17 vs. 6 years). Due to the cross-sectional design of our study, we cannot make assumptions regarding longitudinal changes. On the other hand, since our data are comparable with that of Bergeron et al. [14] with a median follow-up time of 6 years, we may assume that most of the cases with BOS do occur within the first few years after allo-HSCT. This is in line with reports from earlier studies [38, 39]. Although we do not know if the occurrence of pulmonary sequelae found in our study will increase or remain unchanged - or even regress - in the future, we think it is unlikely that the findings on HRCT and the impairments in pulmonary function will change in a clinically significant degree since they were - in general - of a mild nature. However, this assumption may be challenged by new biological research focusing the cellular processes of aging and their association with the premature development of age-related diseases seen in cancer survivors [40]. Since long-term comorbidities observed in cancer survivors seem to mimic the phenotypes of aging, they may be caused by some kind of interaction between therapeutic exposures and the underlying biology of aging [40].

One strength of the present study is the single-center, national patient cohort, uniformly treated according to standardized national protocols. Also, at follow-up, all medical tests were undertaken at the same site and carried out by a limited number of highly selected and experienced staff dedicated to the study, and the same type of equipment was used for, respectively, HRCT and pulmonary function testing. Another strength is an age- and gender-matched group of healthy controls. HRCT was applied in all survivors, not only those with diagnosed or suspected pulmonary complications, which has been the choice in other studies $[16,37]$. From a research point of view, we obviously would have liked to have HRCT results also for the healthy controls, but since it was considered unethical to expose healthy subjects to irradiation for study purposes, the controls were used for comparing pulmonary function data only. Since we aimed to investigate late pulmonary sequelae in very long-term survivors, the long observation time (median 17 years) may be seen as a strength. However, very long observation time also implies survival bias. In our study, $45 \%$ of the alloHSCT patients were deceased at the time of survey. Since national legislation prevents access to information on individual causes of death, we do not know to what extent pulmonary complications may have affected mortality. In a recent long-term study of mortality including almost 4,500 allo-HSCT survivors, Wong et al. [41] reported that if a patient survives the first 2 years after allo-HSCT, the 5 -year survival rates the next 15 years exceed $85 \%$. Furthermore, they found that cGVHD accounted for increasingly fewer deaths among long-term survivors and also a relatively stable mortality rate for pulmonary cause of death throughout the years [41]. Another weakness is the lack of pretreatment data that prevents analysis of longitudinal changes. The patients' age at diagnosis ranged from 0.3 to 30 years. Although pulmonary function testing had not been routinely carried out prior to allo-HSCT and about one-third of the patients would have been too 
young to obtain reliable tests, it might have been possible to track down baseline pulmonary function data from a subset of the patients. Pretreatment HRCT, on the other hand, was not accessible for any patient since it had not been part of the initial routine before allo-HSCT workup for any of the survivors. Furthermore, the cross-sectional design of the study does not allow us to study causal relationships, just describe associations. In a very long-term follow-up study, we think an attendance rate of $66 \%$ is satisfactory. However, we cannot rule out that nonresponse bias may have affected the external validity and generalizability of the results since the nonresponders comprised more males, were younger at the time of treatment, and had shorter observation time. In conclusion, after a median 17 years of observation, allo-HSCT survivors had significantly lower pulmonary function than age- and gender-matched healthy controls and nearly half of them had pathological findings on HRCT. The survivors were young adults (median age 35 years), and longitudinal data are needed to determine if their pulmonary sequelae will remain stable or progress throughout their adulthood. We therefore recommend lifelong monitoring of pulmonary function in allo-HSCT survivors. Pulmonary function testing is cost-effective, readily available, safe, and easy to perform. HRCT may provide valuable additional information and may be indicated for clinical reasons in selected patients. However, HRCT is still a cumbersome and expensive method, and it is also poorly suited for surveillance due to radiation exposure.

\section{Acknowledgment}

The authors would like to thank David Swanson, $\mathrm{PhD}$, for his guidance in statistical analysis.

\section{Statement of Ethics}

The study was approved by the Regional Committee for Medical and Health Research Ethics (2014/370) and the Data Protection Officer at Oslo University Hospital. Written informed consent was obtained from all participants.

\section{Conflict of Interest Statement}

The authors have no conflicts of interest to declare.

\section{Funding Sources}

The study was partially financed by the Norwegian Extra Foundation for Health and Rehabilitation, Project No. 2013-2-230, and the South-Eastern Norway Regional Health Authority, Norway, Grant No. 2015084.

\section{Author Contributions}

M.B.L., T.M.A., L.B., and E.R. designed the study. O.H.M., P.P.D., K.F., and H.M. collected the data. O.H.M., P.P.D., and L.I.S. participated in data analysis. O.H.M., L.I.S., T.M.A., J.K., and M.B.L. interpreted the results and drafted the manuscript. The final version was approved by all authors.

\section{Data Availability Statement}

All data generated or analyzed during this study are included in this article and its supplementary material files. Further enquiries can be directed to the corresponding author.

\section{References}

1 Passweg JR, Baldomero H, Bader P, Bonini C, Cesaro S, Dreger P, et al. Hematopoietic stem cell transplantation in Europe 2014: more than 40000 transplants annually. Bone Marrow Transplant. 2016;51(6):786-92.

2 Majhail NS. Long-term complications after hematopoietic cell transplantation. Hematol Oncol Stem Cell Ther. 2017;10(4):220-7.

3 Haider S, Durairajan N, Soubani AO. Noninfectious pulmonary complications of haematopoietic stem cell transplantation. Eur Respir Rev. 2020;29(156):190119.

4 Li L, Mok H, Jhaveri P, Bonnen MD, Sikora AG, Eissa NT, et al. Anticancer therapy and lung injury: molecular mechanisms. Expert Rev Anticancer Ther. 2018;18(10):1041-57.
5 Abugideiri M, Nanda RH, Butker C, Zhang C, Kim S, Chiang KY, et al. Factors influencing pulmonary toxicity in children undergoing allogeneic hematopoietic stem cell transplantation in the setting of total body irradiationbased myeloablative conditioning. Int J Radiat Oncol Biol Phys. 2016;94(2):349-59.

6 Freycon F, Casagranda L, Trombert-Paviot B. The impact of severe late-effects after $12 \mathrm{~Gy}$ fractionated total body irradiation and allogeneic stem cell transplantation for childhood leukemia (1988-2010). Pediatr Hematol Oncol. 2019;36(2):86-102.

7 Cooke KR, Luznik L, Sarantopoulos S, Hakim FT, Jagasia M, Fowler DH, et al. The biology of chronic graft-versus-host disease: a task force report from the National Institutes of Health consensus development project on criteria for clinical trials in chronic graft-versus-host disease. Biol Blood Marrow Transplant. 2017;23(2):211-34.

8 Sakaida E, Nakaseko C, Harima A, Yokota A, Cho R, Saito Y, et al. Late-onset noninfectious pulmonary complications after allogeneic stem cell transplantation are significantly associated with chronic graft-versus-host disease and with the graft-versus-leukemia effect. Blood. 2003;102(12):4236-42.

9 Bergeron A. Late-onset noninfectious pulmonary complications after allogeneic hematopoietic stem cell transplantation. Clin Chest Med. 2017;38(2):249-62. 
10 Jagasia MH, Greinix HT, Arora M, Williams KM, Wolff D, Cowen EW, et al. National Institutes of Health consensus development project on criteria for clinical trials in chronic graft-versus-host disease: I. The 2014 Diagnosis and Staging Working Group report. Biol Blood Marrow Transplant. 2015;21(3):389401.e1.

11 Verleden GM, Glanville AR, Lease ED, Fisher AJ, Calabrese F, Corris PA, et al. Chronic lung allograft dysfunction: definition, diagnostic criteria, and approaches to treatment-A consensus report from the Pulmonary Council of the ISHLT. J Heart Lung Transplant. 2019; 38(5):493-503.

12 Schlemmer F, Chevret S, Lorillon G, De Bazelaire C, Peffault de Latour R, Meignin V, et al. Late-onset noninfectious interstitial lung disease after allogeneic hematopoietic stem cell transplantation. Respir Med. 2014; 108(10):1525-33.

13 Higo H, Miyahara N, Taniguchi A, Maeda Y, Kiura K. Cause of pleuroparenchymal fibroelastosis following allogeneic hematopoietic stem cell transplantation. Respir Investig. 2019;57(4):321-4.

14 Bergeron A, Chevret S, Peffault de Latour R, Chagnon K, de Margerie-Mellon C, Riviere F, et al. Noninfectious lung complications after allogeneic haematopoietic stem cell transplantation. Eur Respir J. 2018;51(5):1702617.

15 Kloth C, Thaiss WM, Hetzel J, Bier G, Wirths $\mathrm{S}$, Nikolaou K, et al. Results of quantitative chest-CT in chronic pulmonary graft-vs.-host disease (cGvHD) 3 years after allogeneic stem cell transplantation. J Thorac Dis. 2017;9(8): 2521-7.

16 Gunn ML, Godwin JD, Kanne JP, Flowers ME, Chien JW. High-resolution CT findings of bronchiolitis obliterans syndrome after hematopoietic stem cell transplantation. J Thorac Imaging. 2008;23(4):244-50.

17 Cheng GS, Selwa KE, Hatt C, Ram S, Fortuna $\mathrm{AB}$, Guerriero M, et al. Multicenter evaluation of parametric response mapping as an indicator of bronchiolitis obliterans syndrome after hematopoietic stem cell transplantation. Am J Transplant. 2020;20(8):2198-205.

18 Diep PP, Melberg HO, Brinch L, Buechner J, Floisand Y, Gedde-Dahl T, et al. Cost-utility of allogeneic hematopoietic stem cell transplantation in Norway. Bone Marrow Transplant. 2018;53(5):657-60.

19 Pathak M, Diep PP, Lai X, Brinch L, Ruud E, Drolsum L. Ocular findings and ocular graftversus-host disease after allogeneic stem cell transplantation without total body irradiation. Bone Marrow Transplant. 2018;53(7): 863-72.
20 Myrdal OH, Diep PP, Ruud E, Brinch L, Massey RJ, Edvardsen E, et al. Determinants of cardiorespiratory fitness in very long-term survivors of allogeneic hematopoietic stem cell transplantation: a national cohort study. Support Care Cancer. 2021;29(4):1959-67.

21 Massey RJ, Diep PP, Ruud E, Burman MM, Kvaslerud AB, Brinch L, et al. Left ventricular systolic function in long-term survivors of allogeneic hematopoietic stem cell transplantation. JACC CardioOncology. 2020;2(3):46071.

22 Macintyre N, Crapo RO, Viegi G, Johnson DC, van der Grinten CP, Brusasco V, et al. Standardisation of the single-breath determination of carbon monoxide uptake in the lung. Eur Respir J. 2005;26(4):720-35.

23 Wanger J, Clausen JL, Coates A, Pedersen OF, Brusasco V, Burgos F, et al. Standardisation of the measurement of lung volumes. Eur Respir J. 2005;26(3):511-22.

24 Quanjer PH, Stanojevic S, Cole TJ, Baur X, Hall GL, Culver BH, et al. Multi-ethnic reference values for spirometry for the 3-95-yr age range: the global lung function 2012 equations. Eur Respir J. 2012;40(6):1324-43.

25 Stanojevic S, Graham BL, Cooper BG, Thompson BR, Carter KW, Francis RW, et al. Official ERS technical standards: global lung function initiative reference values for the carbon monoxide transfer factor for Caucasians. Eur Respir J. 2017;50(3):1700010.

26 Quanjer PH, Tammeling GJ, Cotes JE, Pedersen OF, Peslin R, Yernault JC. Lung volumes and forced ventilatory flows. Report Working Party Standardization of lung function tests, European Community for Steel and Coal. Official statement of the European Respiratory Society. Eur Respir J Suppl. 1993;16:5-40.

27 Pellegrino R, Viegi G, Brusasco V, Crapo RO, Burgos F, Casaburi R, et al. Interpretative strategies for lung function tests. Eur Respir J. 2005;26(5):948-68.

28 Hansell DM, Bankier AA, MacMahon $\mathrm{H}$, McLoud TC, Müller NL, Remy J. Fleischner Society: glossary of terms for thoracic imaging. Radiology. 2008;246(3):697-722.

29 Bondeelle L, Gras J, Michonneau D, Houdouin V, Hermet E, Blin N, et al. Pleuroparenchymal fibroelastosis after allogeneic hematopoietic stem cell transplantation. Bone Marrow Transplant. 2020;55(5):982-6.

30 Sorensen PG, Rossing N, Rorth M. Carbon monoxide diffusing capacity: a reliable indicator of bleomycin-induced pulmonary toxicity. Eur J Respir Dis. 1985;66(5):333-40.

31 Cooper JA, Matthay RA. Drug-induced pulmonary disease. Dis Mon. 1987;33(2):61-120.
32 Leo F, Solli P, Spaggiari L, Veronesi G, de Braud F, Leon ME, et al. Respiratory function changes after chemotherapy: an additional risk for postoperative respiratory complications? Ann Thorac Surg. 2004;77(1):260-5; discussion 265.

33 Stenehjem JS, Smeland KB, Murbraech K, Holte H, Kvaloy S, Wethal T, et al. Diffusing capacity impairment is prevalent in longterm lymphoma survivors after high-dose therapy with autologous stem cell transplantation. Bone Marrow Transplant. 2017;52(4): 646-9.

34 Myrdal OH, Kanellopoulos A, Christensen JR, Ruud E, Edvardsen E, Kongerud J, et al. Risk factors for impaired pulmonary function and cardiorespiratory fitness in very longterm adult survivors of childhood acute lymphoblastic leukemia after treatment with chemotherapy only(). Acta Oncol. 2018;57(5): 658-64.

35 Landman Y, Stemmer SM, Sulkes A, Neiman V, Granot T, Hendler D, et al. Prospective long-term follow-up of pulmonary diffusion capacity reduction caused by dose-dense chemotherapy in patients with breast cancer. J Oncol. 2019;2019:2584859.

36 Konen E, Gutierrez C, Chaparro C, Murray $\mathrm{CP}$, Chung T, Crossin J, et al. Bronchiolitis obliterans syndrome in lung transplant recipients: can thin-section CT findings predict disease before its clinical appearance? Radiology. 2004;231:467-73.

37 Arakawa H, Webb WR. Air trapping on expiratory high-resolution $\mathrm{CT}$ scans in the absence of inspiratory scan abnormalities: correlation with pulmonary function tests and differential diagnosis. AJR Am J Roentgenol. 1998;170:1349-53.

$38 \mathrm{Au}$ BK, Au MA, Chien JW. Bronchiolitis obliterans syndrome epidemiology after allogeneic hematopoietic cell transplantation. Biol Blood Marrow Transplant. 2011;17(7):10728.

39 Vieira AG, Funke VA, Nunes EC, Frare R, Pasquini R. Bronchiolitis obliterans in patients undergoing allogeneic hematopoietic SCT. Bone Marrow Transplant. 2014;49(6): $812-7$.

40 Cupit-Link MC, Kirkland JL, Ness KK, Armstrong GT, Tchkonia T, LeBrasseur NK, et al. Biology of premature ageing in survivors of cancer. ESMO Open. 2017;2(5):e000250.

41 Wong FL, Teh JB, Atencio L, Stiller T, Kim H, Chanson D, et al. Conditional survival, causespecific mortality, and risk factors of late mortality after allogeneic hematopoietic cell transplantation. J Natl Cancer Inst. 2020; 112(11):1153-61. 